\title{
Use of Calretinin, CD56, and CD34 for Differential Diagnosis of Schwannoma and Neurofibroma
}

\author{
Ji Young Park · Hoon Park ${ }^{1}$ \\ Nam Jo Park · June Sik Park ${ }^{2}$ \\ Hyun-Jung Sung ${ }^{3}$. Sang Sook Lee \\ Department of Pathology, Keimyung University \\ School of Medicine, Daegu; 'Department of \\ Otolaryngology, Korea Cancer Center Hospital, \\ Seoul; '2Department of Otolaryngology, \\ Kyungpook National University School of \\ Medicine; ${ }^{3}$ Department of Pathology, Catholic \\ University of Daegu School of Medicine, Daegu, \\ Korea
}

Received: October 11, 2010

Accepted: October 21, 2010

\section{Corresponding Author}

Sang Sook Lee, M.D.

Department of Pathology, Keimyung University

School of Medicine, 194 Dongsan-dong, Jung-gu, Daegu 700-712, Korea

Tel: +82-53-580-3811, +82-53-250-7922

Fax: +82-53-250-7211

E-mail: sangsook@dsmc.or.kr
Background: It is important to differentiate between schwannomas and neurofibromas for the cases in which the histopathologic features overlap. Depending on the tumor type, surgeons can decide on a treatment method and whether to preserve or sacrifice the nerve; the possibility of malignant transformation in the case of neurofibromas also needs to be considered. Methods: We studied 101 cases of schwannoma and 103 cases of neurofibroma. All the hematoxylin and eosin slides for these cases were reviewed, and tissue microarrays were prepared from the representative areas. Immunohistochemical analysis was performed using antibodies for S-100 protein, calretinin, CD56 and CD34. Results: All the tumors except 3 neurofibromas were positive for the S-100 protein. Calretinin was found in $26.7 \%$ of the schwannomas $(27 / 101)$, but it was not found in any of the neurofibromas. CD56 was positive in $77.2 \%$ of the schwannomas $(78 / 101)$ and in $9.8 \%$ of the neurofibromas (10/102). CD34 was positive in $42.5 \%$ of the schwannomas $(43 / 101)$ and in $80.2 \%$ of the neurofibromas (81/101). Statistically, calretinin was significantly specific for schwannomas $(p<0.001)$ and CD56 was also sensitive for these tumors $(p<0.001)$. On the other hand, a CD34 expression seemed highly sensitive $(p<0.001)$ for neurofibromas. Conclusions: We concluded that combined immunohistochemical analysis for calretinin, CD56, and CD34 may be very useful for differentiating schwannomas from neurofibromas.

Key Words: Schwannoma; Neurofibroma; Immunohistochemistry; Diagnosis, differential
Schwannomas and neurofibromas are the 2 most common types of benign neoplasms derived from the peripheral nerve. ${ }^{1}$ Typically, schwannomas are smooth and well encapsulated; they often grow eccentrically to the nerve from which they arise (Fig. $1 \mathrm{~A}-\mathrm{C}) .^{2}$ These tumors are composed of spindle cells with biphasic architecture that is characterized by organized cellular areas that often display nuclear palisading (Antoni $A$ area) and paucicellular areas (Antoni B area). ${ }^{1,2}$ Neurofibromas are typically grayish tan and less circumscribed than schwannomas (Fig. 1D-F). ${ }^{1,2}$ They show a mixed population of cells, with a predominance of Schwann cells admixed with perineurial-like cells and fibroblasts. ${ }^{2}$ Although schwannomas and neurofibromas are generally not difficult to differentiate by standard light microscopy, in a few cases, there can be considerable morphologic overlap between them. Making the distinction between schwannomas and neurofibromas is very important for a surgeon to be able to determine the choice of operative procedure during intervention. ${ }^{3}$ Schwannomas arise from the nerve lining and they can be surgically removed without sacrificing the nerve. Yet for most neurofibromas, the nerve is a part of the mass, and surgery includes resection and subsequent nerve grafting to preserve and restore function. ${ }^{4}$ In addition, neurofibromas show a small, but non-negligible potential for malignant transformation and they are associated substantially more often with von Recklinghausen disease or neurofibromatosis type 1 (NF-1) than schwannomas. ${ }^{3}$ Several special stains and immunohistochemical markers, including S-100 protein, glial fibrillary acidic protein (GFAP), epithelial membrane antigen (EMA), factor XIIIa, Leu-7, myelin basic protein and Glut-1, have been studied for their ability to differentiate these 2 entities, and they have shown varying sensitivities and specificities. ${ }^{1,2,5-7}$ However, the use of these markers alone or in combination may be inadequate because the 2 entities occasionally show cytomorphologic and immunohistochemical overlap. ${ }^{1,2}$

In the present study, we examined 204 typical cases of schwannomas and neurofibromas by conducting immunohistochemi- 
cal staining for calretinin, CD56, CD34, EMA, S-100 protein and neurofilament protein. We evaluated and compared the expressions of these immunohistochemical markers to arrive at a reliable and useful method for differentiating between schwannomas and neurofibromas.

\section{MATERIALS AND METHODS}

We selected 204 samples of formalin-fixed, paraffin-embedded tissue blocks of schwannomas (101 cases) and neurofibro-
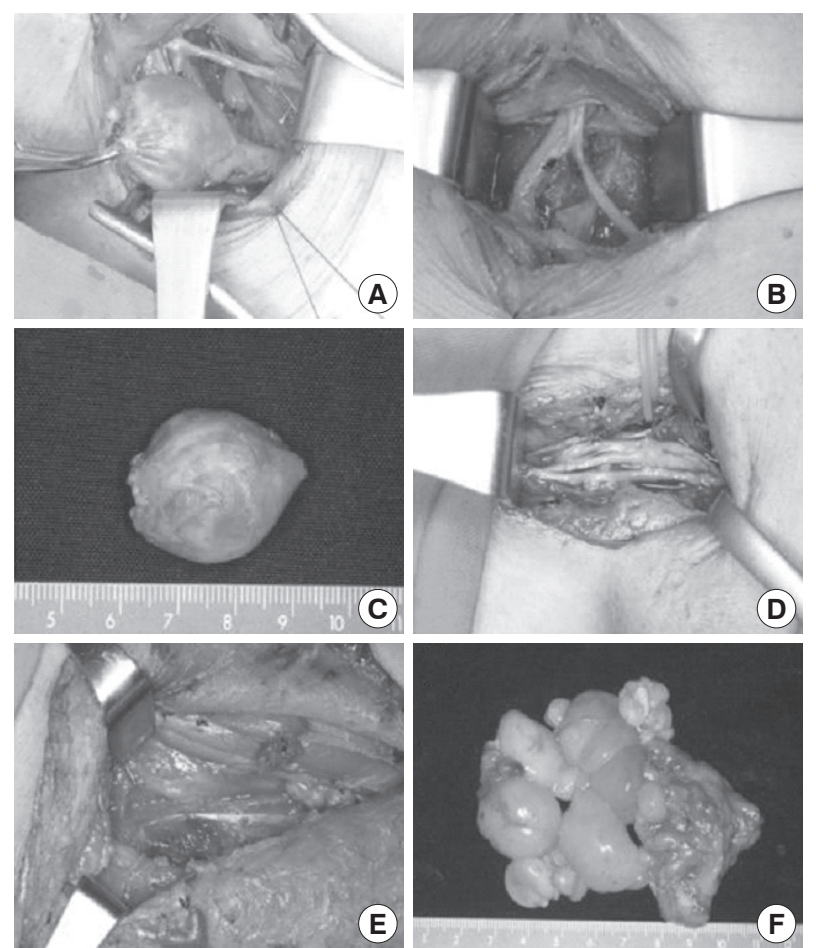

Fig. 1. Intraoperative photos of schwannomas (A-C) and neurofibromas (D-F). A well-encapsulated mass arising from a parent nerve $(A)$ and the preserved parent nerve $(B)$. Excision of the mass $(C)$ in the same schwannoma patient. In the neurofibroma photos, a diffusely enlarged nerve plexus can be seen in 2 patients $(D, E)$; an excised neurofibroma (F) from the latter patient. mas (103 cases) from the archives of the Pathology Department of Keimyung University Hospital, Daegu. Hematoxylin-eosin stained sections of all the samples were available for review, and 2 pathologists and a research worker confirmed the diagnoses. From each case of schwannoma and neurofibroma, two 5.0-mm cores and one 5.0-mm core, respectively, were obtained from the representative areas to make the tissue microarrays with a tissue arrayer. From each tissue microarray block, $4 \mu \mathrm{m}$-thick sections were cut, deparaffinized, rehydrated and quenched with hydrogen peroxide. The sections were stained with immunohistochemical markers for S-100 protein, EMA, neurofilament protein, calretinin, CD56 and CD34 (Table 1). The Labvision kit (Thermo Scientific ${ }^{\circledR}$, Fremont, CA, USA) was used with highmolecular-weight polymers labeled with secondary antibodies and horseradish peroxidase to obtain highly sensitive reactivity. The sections were visualized with diaminobenzidine and counterstained with hematoxylin. Appropriate positive and negative control sections were also used.

The results were evaluated using semi-quantitative scales of the staining intensity and extent. The cases were classified into the negative group (0) when the number of cells stained was less than $5 \%$ and into the positive group when the number of cells stained exceeded 5\%. Immunoreactivity was evaluated as the staining intensities in the positive group (1, weak; 2 , moderate; 3 , strong). For simplicity, the staining intensity values were grouped into 2 categories: negative ( 0 or 1 ) and positive ( 2 or 3). The statistical differences of the immunohistochemical expressions between the schwannomas and neurofibromas were analyzed using Fisher's exact test. Results with p-values $<0.05$ were considered statistically significant. The SPSS ver. 17.0 (SPSS Inc., Chicago, IL, USA) was used for the statistical analysis.

\section{RESULTS}

We evaluated 101 cases of schwannomas and 103 cases of neurofibromas in this study. The schwannoma patients included

Table 1. The antibodies and conditions used in the study

\begin{tabular}{lllc}
\hline Antibody & Clone & Source & Dilution \\
\hline S-100 protein & Polyclonal & BioGenex, San Ramon, CA, USA & $1: 3,000$ \\
EMA & Monoclonal & Dako, Glostrup, Denmark & $1: 400$ \\
Neurofilament protein & Monoclonal & Dako, Carpinteria, CA, USA & $1: 1,000$ \\
Calretinin & Polyclonal & Zymed, San Francisco, CA, USA & $1: 400$ \\
CD56 & Monoclonal & Zymed & $1: 400$ \\
CD34 & Monoclonal & Thermo Scientific, Fremont, CA, USA & $1: 400$ \\
\hline
\end{tabular}

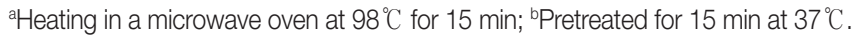

EMA, epithelial membrane antigen. 
66 females and 35 males and their ages ranged from 14 to 72 years, with mean and median ages of 46.8 and 49 years, respectively. Among the neurofibroma patients, there were 63 females and 40 males. The patients' ages ranged from 1 to 70 years, with mean and median ages of 33.6 and 30 years, respectively.

The schwannomas originated from soft tissues ( 43 cases), including the head and neck areas (14), upper extremity (11), lower extremity (8), chest wall (8), retropancreatic area (1) and presacral area (1), followed by the cerebropontine angle of the brain (27), the spinal cord (16), peripheral nerves (7), the mediastinum (5), the retroperitoneum (2) and the pleura (1). The peripheral nerves (7 cases) included the median (3), ulnar (1), cervical (1), trigeminal (1), and common peroneal nerves (1). The neurofibromas were classified into the skin (33 cases) and extra-skin areas (70 cases). The neurofibromas in the latter group developed in the soft tissues of the head and neck areas (37), trunk (14), upper extremity (5), buttock (3), mediastinum (3), lower extremity (2), spinal cord (2), abdominal cavity (1), tongue (1), parotid gland (1) and peripheral nerve (1). Of the 103 neurofibroma samples, 26 had to be excluded from EMA analysis because during processing for EMA immunohistochemical staining, the samples were damaged with loss of arrangement, excessive pretreatment and over-trimming of artifacts. The clinicopathologic reports showed that 22 cases of neurofibromas, but only 1 case of schwannoma was associated with von Recklinghaugen disease or neurofibromatosis type I.

As shown in Table 2, all the schwannoma cases and 100 of the neurofibroma cases $(97.1 \%)$ were positive for S-100 protein $(\mathrm{p}<0.084)$. Further, 27 schwannomas $(26.7 \%)$ expressed calretinin positivity (Fig. 2). In contrast, all the neurofibromas

Table 2. The immunohistochemical profiles of schwannomas and neurofibromas

\begin{tabular}{lcccccc}
\hline & S-100 & Calretinin & CD56 & CD34 & EMA & NFP \\
\hline Schwannomas (\%) & $101 / 101(100.0)$ & $27 / 101(26.7)$ & $78 / 101(77.2)$ & $43 / 101(42.6)$ & $8 / 101(7.9)$ & $6 / 101(5.9)$ \\
Neurofibromas (\%) & $100 / 103(97.1)$ & $0(0.0)$ & $10 / 102(9.8)$ & $81 / 101(80.2)$ & $1 / 77(1.3)$ & $9 / 103(8.7)$ \\
$\chi^{2}$ (p) & $2.986(0.084)$ & $31.735(0.000)$ & $93.943(0.000)$ & $20.137(0.000)$ & $3.991(0.046)$ & $0.616(0.432)$ \\
\hline
\end{tabular}

EMA, epithelial membrane antigen; NFP, neurofilament protein.
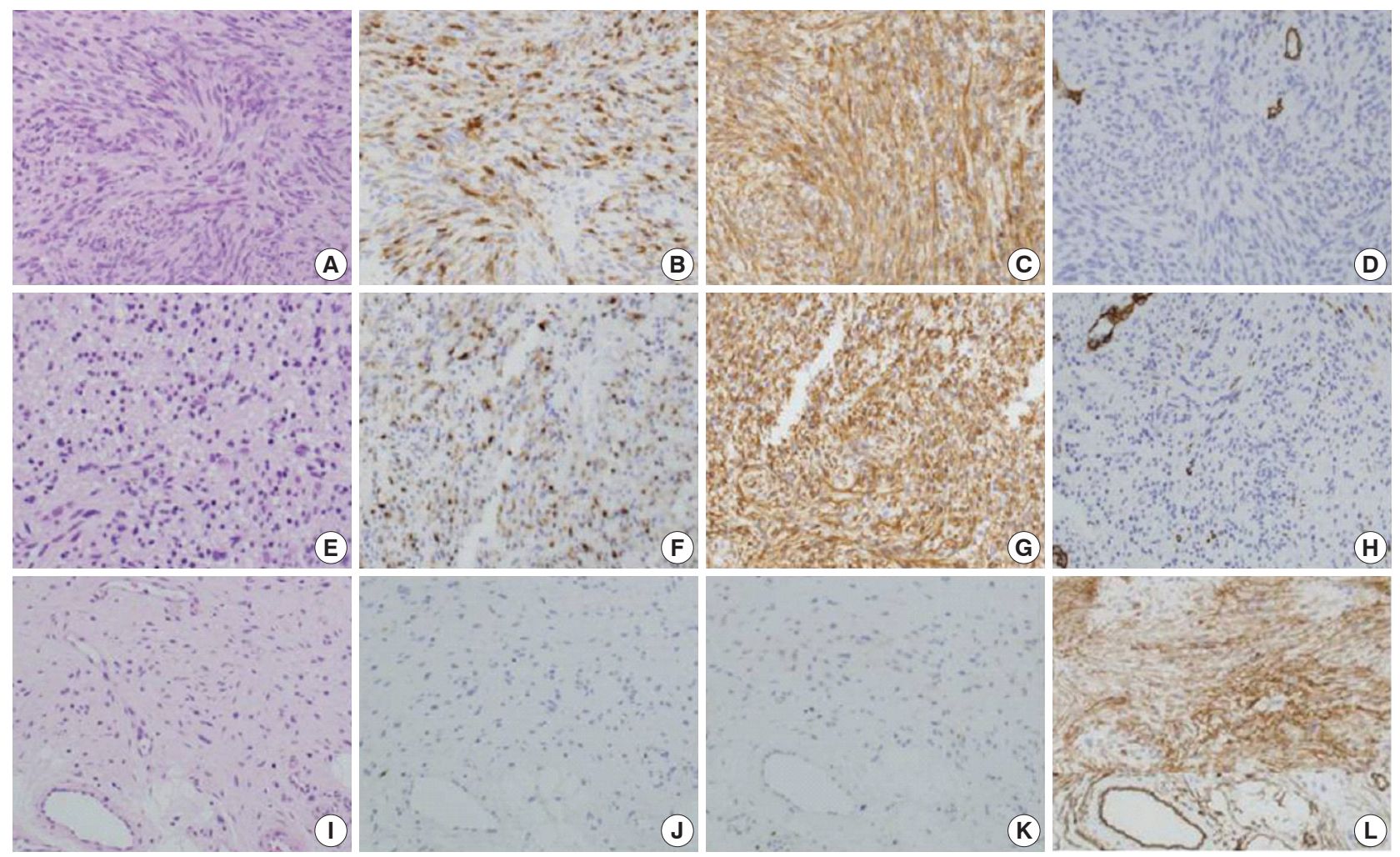

Fig. 2. Representative microscopic features of the Antoni $A$ and Antoni $B$ areas of a schwannoma and a neurofibroma showing the immunoreactivities for calretinin, $C D 56$ and $C D 34$. The Antoni $A(A)$ and Antoni $B(E)$ areas of the schwannoma show strong and diffuse positivity for calretinin $(B, F)$ and CD56 (C, G), but negativity for CD34 (D, H). The neurofibroma (I) expresses CD34 (L), but not calretinin $(J)$ or CD56 (K). 
Table 3. Comparison of the immunohistochemical findings for calretinin, CD56, and CD34 between Antoni A and Antoni B areas of schwannomas

\begin{tabular}{lccc}
\hline & Calretinin & CD56 & CD34 \\
\hline $\begin{array}{c}\text { Schwannomas } \\
\text { (Antoni A) (\%) }\end{array}$ & $29 / 101(28.7)$ & $81 / 101(80.2)$ & $39 / 101(38.6)$ \\
$\begin{array}{c}\text { Schwannomas } \\
\text { (Antoni B) (\%) }\end{array}$ & $25 / 101(24.8)$ & $73 / 101(73.0)$ & $47 / 101(46.5)$ \\
$\chi^{2}(p)$ & $0.404(0.525)$ & $1.453(0.228)$ & $1.296(0.255)$ \\
\hline
\end{tabular}

were immunohistochemically negative for calretinin $(\mathrm{p}<0.001)$ (Fig. 2). With regard to the CD56 expression, 78 schwannomas (77.2\%) were positive, but only 10 neurofibromas (9.8\%) were positive ( $<<0.001$ ) (Fig. 2). Further, 43 schwannomas (42.6\%) and 81 neurofibromas $(73 \%)$ were positive $(\mathrm{p}<0.001)$ for CD34 (Fig. 2), and 8 schwannomas and 1 of 77 neurofibromas (1.3\%) were positive for EMA ( $\mathrm{p}<0.046)$. Only 6 schwannomas $(5.9 \%)$ and 9 neurofibromas $(8.7 \%)$ were positive for neurofilament protein, but these values were not significantly different $(\mathrm{p}<0.432)$.

No significant difference in the calretinin, CD56 and CD34 expressions was found between the Antoni A and Antoni B areas of the schwannomas (Table 3). Similarly, the expression of these markers in the neurofibromas did not differ considerably among the specific tumor locations, the plexiform subtypes (Table 4) and for the presence of von Recklinghausen disease or neurofibromatosis type I.

\section{DISCUSSION}

Schwannomas and neurofibromas arise from the peripheral nerves. ${ }^{1,8,9}$ Schwannomas consist almost exclusively of Schwann cells, while neurofibromas contain several cellular components, including Schwann cells, perineurial-like cells and endoneurial fibroblasts. ${ }^{1,2}$ Schwannomas typically appear as well-encapsulated, firm masses that are tan in color with a variable degree of yellow coloration, and they grow eccentrically to the nerve from which they arise. Although the parent nerve is often grossly apparent, it may not be possible to detect it if the schwannoma is large or the parent nerve is small., ${ }^{2,-12}$ In contrast to schwannomas, neurofibromas show a mixed population of cells, with the predominance of Schwann cells admixed with perineurial-like cells and fibroblasts and interspersed non-neoplastic nerve fibers, collagen fibers and a myxoid matrix. Grossly, they are glistening and grayish tan, they range in consistency from gelatinous to firm and they lack the secondary degenerative changes common to schwannomas. ${ }^{2,9,11,13}$ The diffuse involvement of the
Table 4. Immunohistochemical findings for calretinin, CD56, and CD34, according to the subtype and specific location (skin) of neurofibromas

\begin{tabular}{lccc}
\hline & Calretinin & CD56 & CD34 \\
\hline Neurofibromas & & & \\
$\quad$ Total (\%) & $0 / 103(0.0)$ & $10 / 102(9.8)$ & $81 / 101(80.2)$ \\
$\chi^{2}(p)$ & - & - & $93.943(0.000)$ \\
Plexiform type (\%) & $0 / 26(0.0)$ & $5 / 26(19.2)$ & $24 / 26(92.3)$ \\
$\chi^{2}(p)$ & - & $2.117(0.146)$ & $1.780(0.181)$ \\
Skin (\%) & $0 / 33(0.0)$ & $4 / 33(12.1)$ & $21 / 33(63.6)$ \\
$\chi^{2}(p)$ & - & $2.538(0.111)$ & $2.657(0.103)$ \\
\hline
\end{tabular}

nerves in neurofibromas may make complete resection impossible; such neurofibromas are commonly seen in the orbit, neck, back and inguinal region. ${ }^{11}$

Although schwannomas and neurofibromas are generally not difficult to differentiate microscopically by their pattern of growth and cellular composition, there can be considerable morphologic overlap between them. Nuclear palisading is not always seen in schwannomas and some these schwannomas are potentially difficult to separate from cellular neurofibromas. ${ }^{1}$ Furthermore, schwannomas consisting exclusively of Antoni B areas are sparsely cellular and myxomatous and so they might mimic the histologic appearance of neurofibromas. ${ }^{1,10}$ Colocalized schwannomas and neurofibromas have also been reported, which makes it even more difficult to distinguish between them. ${ }^{13}$

Yet distinguishing between schwannomas and neurofibromas is important because the latter are much more often associated with von Recklinghausen disease or NF-1, and so they show a small but non-negligible potential for malignant transformation. ${ }^{3}$ Further, the surgical management also differs depending on whether the tumor is a schwannoma or neurofibroma. ${ }^{3}$ Schwannomas can be easily removed while preserving nerve contiguity. ${ }^{1,4,14}$ However, most neurofibromas are embedded within the nerve mass, and surgery involves resection and sacrifice of nerve, with subsequent nerve grafting to preserve and restore function. $^{13}$

Several special stains and immunohistochemical markers have been studied for making the differential diagnosis of schwannomas and neurofibromas. ${ }^{1,2}$ The special stains include trichrome, alcian blue and reticular and myelin stains, which can be used to highlight the stromal components such as collagen deposits or stromal mucin. Immunohistochemical stains for S-100 protein, GFAP, EMA, factor XIIIa, Leu-7, myelin basic protein and Glut-1 can help differentiate the tumors, but they have different sensitivities and specificities. ${ }^{1-3,12,15,16} \mathrm{~S}-100$ protein is a preferred marker for identifying cells/tumor/tissue with a nerve origin. Fine et al. ${ }^{1}$ have suggested that calretinin alone or in com- 
bination with S-100 protein is useful for differentiating schwannomas from neurofibromas. However, occasional cases with cytomorphologic and immunohistochemical overlap do exist and so the exact distinction between the 2 tumors is still not very clear.

Calretinin is a calcium-binding protein that belongs to the family of EF-hand proteins, which includes S-100 protein. ${ }^{1} \mathrm{Cal}-$ retinin is primarily expressed in certain types of neurons in the central and peripheral nervous systems. ${ }^{1}$ An extraneuronal expression of calretinin is known to be a marker of human mesothelial cells and mesotheliomas, certain types of ovarian epithelial and stromal cells, some types of ovarian sex-cord stromal tumors, Leydig cell tumors of the testis, adrenal cortical tumors and adenomatoid tumors. ${ }^{1,10,17}$ In this study, the calretinin expression was found to be exclusively confined to schwannomas. None of the neurofibromas showed a positive reaction for calretinin. Therefore, calretinin can be regarded as a highly specific marker for schwannomas.

CD56 is a neural cell adhesion molecule. It is an integral membrane glycoprotein that mediates calcium-independent homophilic cell-cell binding. ${ }^{7,16,18} \mathrm{CD} 56$ is expressed in many normal cells and tissues, including neurons, astrocytes, glia of the cerebral cortex and cerebellum, adrenal cortex (zona glomerulosa) and medulla, human natural killer cells and a subset of $\mathrm{T}$ lymphocytes. ${ }^{7,16,18}$ The soft tissue tumors that often express CD56 include synovial sarcoma, malignant peripheral nerve sheath tumor, schwannoma, rhabdomyosarcoma, leiomyosarcoma, leiomyoma, chondrosarcoma and osteosarcoma. ${ }^{7,16,18}$ In this study, $77.2 \%$ of the schwannomas expressed CD 56 , while only $9.8 \%$ of the neurofibromas expressed it. This result suggested that $\mathrm{CD} 56$ might be a sensitive marker for schwannomas.

$\mathrm{CD} 34$ or the human hematopoietic progenitor cell antigen is expressed by the embryonic cells of the hematopoietic system, including endothelial cells and lymphoid/myelogenous elements. Although CD34 is known to be expressed on vascular tumors, it is also a marker of nerve sheath cells. It has been suggested that the nature of $\mathrm{CD} 34$-positive cells corresponds to that of endoneurial fibroblasts. ${ }^{5,10,19,20}$ In this study, CD34 was expressed by almost 2 times the number of neurofibromas (80.2\%) as that of schwannomas (42.6\%). Therefore, CD34 might be a useful sensitive marker for neurofibromas rather than schwannomas. In this study, calretinin, CD56 and CD34 were found to be statistically significant.

Our results demonstrate that calretinin is highly specific as a marker for schwannomas and CD56 is a sensitive marker of schwannomas. Positivity for both calretinin and CD56 in the S- 100-positive cases is highly suggestive of schwannomas. However, CD34 appears more sensitive for neurofibromas. The clinicopathologic factors, including the Antoni A or Antoni B areas of schwannomas, the tumor locations and subtypes, and the presence of von Recklinghausen disease or NF-1 in neurofibromas do not affect the expression of calretinin, CD56, and CD34. Thus, this study provides strong evidence that combined immunohistochemical staining for calretinin, CD56 and CD 34 in addition to the light microscopic findings can be very useful as a simple, easy and reliable method for differentiating schwannomas and neurofibromas.

\section{REFERENCES}

1. Fine SW, McClain SA, Li M. Immunohistochemical staining for calretinin is useful for differentiating schwannomas from neurofibromas. Am J Clin Pathol 2004; 122: 552-9.

2. Skovronsky DM, Oberholtzer JC. Pathologic classification of peripheral nerve tumors. Neurosurg Clin N Am 2004; 15: 157-66.

3. Nascimento AF, Fletcher CD. The controversial nosology of benign nerve sheath tumors: neurofilament protein staining demonstrates intratumoral axons in many sporadic schwannomas. Am J Surg Pathol 2007; 31: 1363-70.

4. Tsai WC, Chiou HJ, Chou YH, Wang HK, Chiou SY, Chang CY. Differentiation between schwannomas and neurofibromas in the extremities and superficial body: the role of high-resolution and color Doppler ultrasonography. J Ultrasound Med 2008; 27: 161-6.

5. Dabbs DJ. Diagnostic immunohistochemistry. Philadelphia: Churchill Livingstone, 2002; 75-7.

6. Dabbs DJ. Diagnostic immunohistochemistry. Philadelphia: Churchill Livingstone, 2002; 80.

7. Miettinen MM. Diagnostic soft tissue pathology. Philadelphia: Churchill Livingstone, 2003; 53-7.

8. Miettinen MM. Diagnostic soft tissue pathology. Philadelphia: Churchill Livingstone, 2003; 347-60.

9. Weiss SW, Goldblum JR. Enzinger and Weiss's soft tissue tumors. St. Louis: Mosby, 2008; 135.

10. Hirose T, Tani T, Shimada T, Ishizawa K, Shimada S, Sano T. Immunohistochemical demonstration of EMA/Glut1-positive perineurial cells and CD34-positive fibroblastic cells in peripheral nerve sheath tumors. Mod Pathol 2003; 16: 293-8.

11. Rosai J. Rosai and Ackerman's surgical pathology. 9th ed. St. Louis: Mosby, 2004; 2264-9.

12. Weiss SW, Goldblum JR. Enzinger and Weiss's soft tissue tumors. St. Louis: Mosby, 2008; 835-72. 
13. Martinoli C, Bianchi S, Derchi LE. Tendon and nerve sonography. Radiol Clin North Am 1999; 37: 691-711.

14. Spinner RJ, Scheithauer BW, Perry A, Amrami KK, Emnett R, Gutmann DH. Colocalized cellular schwannoma and plexiform neurofibroma in the absence of neurofibromatosis: case report. J Neurosurg 2007; 107: 435-9.

15. Laskin WB, Fetsch JF, Lasota J, Miettinen M. Benign epithelioid peripheral nerve sheath tumors of the soft tissues: clinicopathologic spectrum of 33 cases. Am J Surg Pathol 2005; 29: 39-51.

16. Weiss SW, Goldblum JR. Enzinger and Weiss's soft tissue tumors.
St. Louis: Mosby, 2008; 153.

17. Dabbs DJ. Diagnostic immunohistochemistry. Philadelphia: Churchill Livingstone, 2002; 209-10.

18. Dabbs DJ. Diagnostic immunohistochemistry. Philadelphia: Churchill Livingstone, 2002; 142-3.

19. Miettinen MM. Diagnostic soft tissue pathology. Philadelphia: Churchill Livingstone, 2003; 45-6.

20. Weiss SW, Goldblum JR. Enzinger and Weiss's soft tissue tumors. St. Louis: Mosby, 2008; 143-7. 